\title{
Atomoxetine and circadian gene expression in human dermal fibroblasts from study participants with a diagnosis of attention-deficit hyperactivity disorder
}

\author{
Frank Faltraco $^{1} \cdot$ Denise Palm $^{1} \cdot$ Adriana Uzoni $^{1} \cdot$ Frederick Simon $^{1} \cdot$ Oliver Tucha $^{1} \cdot$ Johannes Thome ${ }^{1}$
}

Received: 12 January 2021 / Accepted: 27 June 2021 / Published online: 17 July 2021

(c) The Author(s) 2021

\begin{abstract}
Atomoxetine (ATO) is a second line medication for attention-deficit hyperactivity disorder (ADHD). We proposed that part of the therapeutic profile of ATO may be through circadian rhythm modulation. Thus, the aim of this study was to investigate the circadian gene expression in primary human-derived dermal fibroblast cultures (HDF) after ATO exposure. We analyzed circadian preference, behavioral circadian and sleep parameters as well as the circadian gene expression in a cohort of healthy controls and participants with a diagnosis of ADHD. Circadian preference was evaluated with German Morningness-Eveningness-Questionnaire (D-MEQ) and rhythms of sleep/wake behavior were assessed via actigraphy. After ex vivo exposure to different ATO concentrations in HDF cultures, the rhythmicity of circadian gene expression was analyzed via qRT-PCR. No statistical significant effect of both groups (healthy controls, ADHD group) for mid-sleep on weekend days, mid-sleep on weekdays, social jetlag, sleep WASO and total number of wake bouts was observed. D-MEQ scores indicated that healthy controls had no evening preference, whereas subjects with ADHD displayed both definitive and moderate evening preferences. ATO induced the rhythmicity of Clock in the ADHD group. This effect, however, was not observed in HDF cultures of healthy controls. Bmall and Per 2 expression showed a significant $\mathrm{ZT} \times$ group interaction via mixed ANOVA. Strong positive correlations for chronotype and circadian genes were observed for Bmall, Cryl and Per 3 among the study participants. Statistical significant different Clock, Bmall and Per3 expressions were observed in HDFs exposed to ATO collected from ADHD participants exhibiting neutral and moderate evening preference, as well as healthy participants with morning preferences. The results of the present study illustrate that ATO impacts on circadian function, particularly on Clock, Bmall and Per2 gene expression.
\end{abstract}

Keywords Atomoxetine · Human dermal fibroblasts · Circadian rhythm

\section{Introduction}

Circadian rhythmicity is a fundamental feature of mammalian physiology that has developed under the continuous evolutionary pressure of energy conservation and efficiency. Evolution has fine-tuned the inner clock to anticipate and respond to several environmental stimuli. In modern technological societies, circadian entrainment is subjected to alterations from artificial lighting as well as lifestyle changes.

Frank Faltraco

frank.faltraco@med.uni-rostock.de

1 Department of Psychiatry and Psychotherapy, University Medical Centre Rostock, Rostock, Gehlsheimer Str. 20, 18147 Rostock, Germany
These alterations lead to the increased development of disorders, e.g. attention-deficit hyperactivity disorder (ADHD), depression, obesity, as well as diabetes (Gerhart-Hines and Lazar 2015).

Attention deficit hyperactivity disorder (ADHD) is one of the most common childhood psychiatric disorders. The disease persists into adulthood in $40 \%$ of children with ADHD (Biederman et al. 2000). ADHD symptoms are characterized by inattention and/or hyperactivity-impulsivity. The disorder is associated with characteristic behavioral difficulties and impairments of day-to-day functioning.

ADHD is associated with circadian rhythm disturbances as well as disturbances in the circadian genes (Clock, Bmall, Per1-3, Cry1-2). Individuals with a diagnosis of ADHD often exhibit disturbances in the circadian day-night rhythm (Rybak et al. 2007). Adults with a diagnosis of ADHD often 
have difficulties falling asleep as well as sleep through the night (Konofal et al. 2010; Philipsen et al. 2006).

Several genome-wide-association-studies (GWASs) have detected associations between chronotype and genotype (Gaspar 2017; Hu et al. 2016; Jones 2016; Kalmbach et al. 2017; Lane 2016). Lane et al. (2016) reported 12 loci associated with chronotype. Some of these loci are in the region of circadian genes (Lane et al. 2016). Jones et al. (2016) reported 16 loci associated with the morningness chronotype. These genes were reported to be involved in circadian rhythm and photoreception (Jones et al. 2016). Hu et al. (2016) found 15 loci associated with morningness. Seven of these loci are near to circadian genes (Hu et al. 2016). Nine of the loci reported in the Lane et al. study and the Jones et al. study were common (Kalmbach et al. 2017).

Atomoxetine (ATO) is a second line pharmacological treatment for ADHD, besides methylphenidate, which sometimes is associated with adverse reactions and intolerance. ATO is a selective noradrenaline reuptake (NET) inhibitor. It increases norepinephrine and dopamine extracellular levels in the prefrontal cortex of rats (Bymaster et al. 2002; Swanson et al. 2006). It has been found to act as an $\mathrm{N}$-methyl-D-aspartate receptor antagonist in rat cortical neurons (Bymaster et al. 2002; Swanson et al. 2006).

The major active metabolite, 4-hydroxyatomoxetine, has been found to have sub-micromolar affinity for opioid receptors, acting as partial agonist of the kappa-opioid receptor (Bymaster et al. 2002; Swanson et al. 2006). Orally administered ATO is rapidly absorbed. First-pass metabolism is dependent on the member of the cytochrome P450CYP2D6 activity, resulting in an absolute bioavailability of 63\% for extensive metabolizers and $94 \%$ for poor metabolizers (Bymaster et al. 2002; Swanson et al. 2006). ATO reaches maximum plasma concentration within about $1-2 \mathrm{~h}$ of administration with the half-life varying widely between individuals, with average range of 4.5-19 h (Bymaster et al. 2002; Swanson et al. 2006). In extensive metabolisers, ATO has a plasma half-life of $5.2 \mathrm{~h}$, while in poor metabolisers, 21.6 h (Bymaster et al. 2002; Swanson et al. 2006).

A study reported that daily methylphenidate and ATO treatment impacts on circadian gene protein expression in the mouse brain (Baird et al. 2013). The protein expression in the hypothalamus of mice effects on PER2 in the nucleus suprachiasmaticus (SCN) as well as on PER1 in the paraventricular nucleus after ATO daily treatment. The same group observed that PER2 expression is effected in the caudate and putamen, while PER1 expression is effected in the ventral tegmental area (Baird et al. 2013). Other studies demonstrated that ATO reduces core symptoms of ADHD and is well tolerated (Michelson 2001; Spencer 2001). In a trial, Griffiths (2018) observed that 6-week treatment with ATO improved ADHD and anxiety symptoms as well as primary cognitive outcomes of response inhibition compared to placebo, in children and adolescents with ADHD. No changes were observed for sustained attention (Griffiths et al. 2018). A recent study in healthy adults demonstrated that ATO modulates reward value (Suzuki et al. 2019). It is proposed that part of the therapeutic profile of ATO may be through circadian rhythm modulation, being able to phase shift the circadian clock in mice (O'Keeffe et al. 2012). Coogan (2019) reported that patients with ADHD using ADHD-medication, methylphenidate and ATO, have lower relative amplitudes of diurnal activity rhythms, lower sleep efficiency and more nocturnal activity than both, healthy controls, and ADHD participants without medication (Coogan et al. 2019).

In this study, we investigate the effects of ATO on circadian rhythm using human dermal fibroblasts (HDFs) derived from ADHD and healthy individuals. Patient-derived HDF cultures have the advantage of being independent to light exposure and express the majority of the same receptors and pathways as neurons, including catecholamine pathways. HDFs cultures are easily established from skin biopsies and provide an advantageous model to study circadian rhythmicity and the influence of drugs on circadian gene expression (Coogan et al. 2019). We successfully established a model based on skin fibroblasts for the investigation of the circadian rhythm, particularly, circadian gene expression. The model was applied for the comparison between healthy controls and two ADHD groups, one group with medication and the other group without medication (Coogan et al. 2019).

The current study examines circadian rhythms at the behavioral and molecular levels in healthy participants and patients with ADHD, after exposure of HDFs to ATO. The goals of the study were (1) to evaluate the circadian preference, behavioral circadian as well as sleep parameters in healthy participants and patients with a diagnosis of ADHD (2) and to examine the molecular levels of clock genes after exposure of primary human-derived dermal fibroblast cultures to ATO.

Based on the assumption of the effectiveness of ATO for the treatment of ADHD, its influence on the expression of circadian genes is hypothesized. Therefore, in this study, the influence of ATO on circadian rhythmicity is investigated in vitro.

\section{Materials and methods}

\section{Participant selection criteria}

Ethical approval for the conduct of the study, including obtaining human dermal biopsy samples, was given by the ethical review committee of Rostock University (Registration-number: A2013-159) and written consent was obtained from each study participant. The study was conducted 
according to the ethical guidelines of the Declaration of Helsinki.

In total, 12 volunteers without a neuropsychiatric diagnosis (healthy controls) and 12 volunteers with attention-deficit hyperactivity disorder (ADHD) participating in the study were recruited via the Department of Psychiatry and Psychotherapy, University Medical Centre Rostock. All ADHD subjects meeting DSM-IV and ICD-10 criteria diagnosed by experienced psychiatrists in advance. The healthy control group was recruited of acquaintances of people involved in the study.

Human dermal fibroblasts (HDF) were obtained from skin biopsies from dorsal forearm from ADHD patients and healthy control volunteers. Only adult individuals, able to give informed consent, were included. Healthy controls without a history of childhood and adult ADHD were matched for sex and age. Patients with more severe psychiatric symptoms were excluded, as were shift workers. Screening for ADHD symptoms was done by assessment of symptoms according to DSM-IV and ICD-10 criteria. Additionally, the following psychometric tests were used to confirm ADHD diagnosis: Wender Utah Rating Scale (WURS-k), SKIDI and II (Structured clinical interview), DIVA 2.0 (Structured diagnostic interview), CAARS (Conners' Adult ADHD Rating Scales) and PSQI (Pittsburgh Sleep Quality Index). The IQ of the healthy control group and volunteers with ADHD diagnosis was measured using the Multiple Choice Word Test (MWT). The chronotype of the participants was determined by the Morning-Eveningness-Questionnaire, German Version (D-MEQ). No special cognitive testing was implemented in the study.

Comorbidities were observed: $16.7 \%$ of participants with ADHD diagnosis has additionally adipositas, $8.3 \%$ has additionally addiction disorder, and $25 \%$ has additionally affective disorder. The remaining participants with ADHD diagnosis have no comorbidities.

The four manuscripts of this special issue dealing with circadian rhythmicity describe unique research questions (Faltraco et al. 2021a, b; Palm et al. 2021). Although some samples have been used for more than one research question, the overall sample composition differs from each other and thus is different for each study. Experiments differ substantially in their conditions, thus, they each investigate unique cellular biochemical pathways.

\section{Actigraphy}

To obtain objective measures of participants' sleep and circadian rhythm function, the rest-activity pattern of participants was recorded using wrist-worn actigraphs (Actiwatch 2, Philips Respironics, USA). Actigraphs were worn on the non-dominant wrist for a period of at least 7 consecutive days. The recording interval of the device was set at 60 -s epochs. Data occurring before the first and after the final midnight of each record were excluded, ensuring at least 6 complete days for each participant, with a complete weekend included in each record.

\section{Tissue isolation and fibroblast cell culture}

Human dermal fibroblasts (HDF) were isolated and cultured as described previously (Takashima 1998). Fibroblasts were cultivated $\left(37{ }^{\circ} \mathrm{C}, 5 \% \mathrm{CO}_{2}\right)$ in Dulbecco's Modified Eagle Medium DMEM (Gibco, Thermo Fisher, UK)/1 mg/ml Liberase TM (Roche, Germany) containing 100 units/ml penicillin, $100 \mu \mathrm{g} / \mathrm{ml}$ streptomycin (Gibco, Thermo Fisher, UK) and $10 \%$ fetal bovine serum FBS (Gibco, Thermo Fisher, UK).

\section{Measurement of cell viability}

Upon confluency of the respective primary fibroblast cell culture from each participant, cells were incubated with $0 \mu \mathrm{M}, 0.2 \mu \mathrm{M}$ and $0.58 \mu \mathrm{M}$ ATO (Atomoxetine hydrochloride, European Pharmacopoeia (EP) Reference Standard, Merck, Germany) in DMEM with 5\% FBS. Following $24 \mathrm{~h}$, cell viability was measured using the Trypan Blue Exclusion Test (Strober 2015).

\section{Measurement of circadian gene expression}

Upon confluency of the respective primary fibroblast cell culture from each participant, eight culture flask replicates were prepared and cells were incubated with either $0.2 \mu \mathrm{M}$ or $0.58 \mu \mathrm{M}$ ATO (Atomoxetine hydrochloride, European Pharmacopoeia (EP) Reference Standard, Merck, Germany) in DMEM with 5\% FBS for $24 \mathrm{~h}$. Cultures without ATO were used as a negative control. The following day, the cells were synchronized with $100 \mathrm{nM}$ dexamethasone (SigmaAldrich, Germany) for $30 \mathrm{~min}$. Samples were harvested every fourth hour after synchronization for a period of $28 \mathrm{~h}$ in GTC lysis buffer (4.5 M guanidinium thiocyanate, $0,5 \%$ sodium- $N$-lauryl sarcosine, $25 \mathrm{mM}$ tri-sodium citrate, $0.1 \mathrm{M}$ betamercaptoethanol) and stored at $-80^{\circ} \mathrm{C}$. mRNA was isolated and purified with RNeasy Plus Mini Kit (Qiagen, Germany) as well as subjected to reverse transcription using the Superscript III First-Strand Synthesis System (Invitrogen, Germany). Gene expression of Clock, Bmall, Cryl, Perl, Per2, and Per3 as well as housekeeping genes, Rpl13A, Rpl19A, GAPDH, was measured by real-time quantitative reverse transcriptase polymerase chain reaction (qRT-PCR) with CFX Connect ${ }^{\mathrm{TM}}$ Real-Time PCR Detection System (Biorad, Germany). All primers were purchased from Eurofins (Alameda, CA). The oligonucleotide sequences are presented in Table 1. The qRT-PCR was performed in 96-well 0.1-ml thin-wall PCR plates (Applied Biosystems) in the 
Table 1 Oligonucleotides for qRT-PCR to measure circadian gene expression

\begin{tabular}{lll}
\hline Gene & Forward primer $\left(5^{\prime}-3^{\prime}\right)$ & Reverse primer $\left(5^{\prime}-3^{\prime}\right)$ \\
\hline Clock & CCAGCAGTTTCATGAGATGC & GAGGTCATTTCATAGCTGAGC \\
Pmal1 & AAGGATGGCTGTTCAGCACATGA & CAAAAATCCATCTGCTGCCCTG \\
Per 2 & TGGGGACAACAGAACAGAGAA & AGGACACTCCTGCGACCA \\
Per 3 & GTATCCATTCATGCTGGGCT & TCGTTTGAACTGCGGTGAC \\
Cry 1 & TCAGTGTTTGGTGGAAGGAA & TCTGGGTCAGCAGCTCTACA \\
RPL13a & CACGAATCACAAACAGACGG & TACATCCTGGACCCCTGGT \\
RPL19a & GCCAGAAATGTTGATGCCTT & AGATGGCGGAGGTGCAG \\
GAPDH & GTGGCAAGAAGAAGGTCTGG & GCCCATCTTTGATGAGCTTC \\
\hline
\end{tabular}

CFX Connect ${ }^{\mathrm{TM}}$ Real-Time PCR Detection System (Biorad, München, Germany). Each $20 \mu$ reaction contained $10 \mu \mathrm{l}$ PerfeCTa SYBR Green Master Mix (Quantabio, Munich, Germany), $200 \mathrm{nM}$ gene-specific forward and reverse primer mix (Eurofins, Alameda, CA) and 20 ng template DNA. For normalization of the expression levels of genes of interest, the geometrical mean of expression level of housekeeping genes Rpl13A, Rpl19A, GAPDH from the same sample was used (Mane et al. 2008). The primer efficiency (between 1.93 and 2.00) was evaluated using the RegLinePCR v 11.0 (Heart Failure Research Center). Data were analyzed using the $\Delta \Delta \mathrm{Ct}$ method (Livak and Schmittgen 2001). The values were normalized to corresponding individual averages.

\section{Statistical methods}

To determine the best-fitting linear harmonic regression, circadian gene expression data were tested for significant circadian rhythmicity, using CircWave v.1.4 software (generated by Dr. Roelof Hut; www.euclock.org). The data were set to an assumed period of $24-h$ and with $\alpha$ set at 0.05 . The center-of-gravity of each best-fitting waveform in CircWave was used as the circadian acrophase, and the associated estimation error was used as the SD. Inferential statistics were carried out in SPSS (IBM Corporation). Actigraphic data were analyzed via MANCOVAs, with age, sex and in some cases, ADHD symptom severity included in the model as covariates.

qRT-PCR clock gene data were analyzed via ANOVA and mixed between-within ANOVAs. One-way ANOVA was used to assess differences of clock gene expression levels among chronotype groups. Associations between clock gene expression and chronotype obtained from healthy controls and ADHD participants were studied by Spearman's rank order correlation. For all inferential tests, $P<0.05$ was used to indicate a statistically significant groupwise difference. Sample sizes were calculated via GPower 3.1 software; for correlations the assumptions used were significance level of $\alpha=0.05$ and the power of 0.8 for 2 groups (ADHD, HC) with 3 measures $(0 \mu \mathrm{M}, 0.2 \mu \mathrm{M}$ and $0.58 \mu \mathrm{M}$ ATO). Although research in this field is generally scarce, we assumed that the influence of ATO on the circadian gene expression will have an effect size $d^{\prime}=0.5$, returning a required total sample size of 21. Taking into consideration, an expected drop-out rate, $n=12$ participants were allocated to each group.

Data were analyzed via time series statistics adequately powered by 12 samples each, which in this statistical model is mathematically sufficient and thus representative (Menet et al. 2012; Thaben and Westermark 2016).

\section{Results}

\section{Demographic data}

Human dermal fibroblasts (HDFs) were obtained via skin biopsy from healthy controls (HC) (4 men, 8 women; $41.50 \pm 14.04$ years, mean \pm SD; BMI: $25.87 \pm 5.42 \mathrm{~kg} / \mathrm{m}^{2}$, mean $\pm \mathrm{SD}$ ) and volunteers with attention-deficit hyperactivity disorder (ADHD) (7 men, 5 women; $42.00 \pm 12.88$ years, mean \pm SD; BMI: $25.86 \pm 3.83 \mathrm{~kg} / \mathrm{m}^{2}$, mean $\left.\pm \mathrm{SD}\right)$. All participants completed the Multiple-Choice Word Test (IQ score: HC: $110.25 \pm 9.32$, mean \pm SD; ADHD participants: $109.42 \pm 11.66$, mean $\pm S D$, n.s), MorningnessEveningness-Questionnaire, German Version (D-MEQ Score: HC: $58.83 \pm 8.97$, mean \pm SD; ADHD participants:: $48.50 \pm 14.92$, mean \pm SD, n.s.) and Wender Utah Rating Scale, German Short Version (WURS-k Score: HC: $7.17 \pm 8.19$, mean \pm SD; ADHD participants: $37.08 \pm 16.47$, mean $\pm \mathrm{SD}, p<0.001)$. The demographic data are presented in Table 2.

There were no significant differences in age, BMI, IQ, D-MEQ or gender across the two study groups. D-MEQ scores indicated that $\mathrm{HC}$ had no evening preference, whereas ADHD patients displayed both definitive and moderate evening preferences. 
Table 2 Demographic data

\begin{tabular}{lll}
\hline Demographic data & $\begin{array}{l}\text { Healthy controls } \\
n=12\end{array}$ & $\begin{array}{l}\text { ADHD } \\
n=12\end{array}$ \\
\hline Age & $41.50 \pm 14.04$ years & $42.00 \pm 12.88$ years \\
Female & $8(66.7 \%)$ & $5(35.4 \%)$ \\
BMI & $25.87 \pm 5.42$ & $25.86 \pm 3.83$ \\
IQ-Score & $110.25 \pm 9.32$ & $109.42 \pm 11.66$ \\
D-MEQ & $58.83 \pm 8.97$ & $58.83 \pm 8.97$ \\
Chronotype & $7(58.3 \%)$ Neutral type & $3(25.0 \%)$ Neutral \\
& $3(25.0 \%)$ Moderate & type \\
& morning type & $5(41.7 \%)$ Moderate \\
& $2(16.7 \%)$ Definitive & morning type \\
& morning type & $2(16.7 \%)$ Moderate \\
& & evening type \\
& & $2(16.7 \%)$ Defini- \\
& & tive evening type \\
WURS-k-Score & $7.17 \pm 8.19 * * *$ & $37.08 \pm 16.47 * * *$ \\
\hline
\end{tabular}

$* * * p<0.001$

\section{Actigraphy}

Measures from the non-parametric circadian rhythm analysis were analyzed between both groups, healthy controls and ADHD participants, in a MANCOVA. Age and sex were chosen as co-variates. No statistically significant effect of group was observed (Pillai's trace $=0.205$; $F=0.643 ; p=0.695$; partial ETA squared $=0.205$ ). Bonferroni post hoc correction demonstrated no significant difference for mid-sleep on weekend days $(p=0.774)$, midsleep on weekdays $(p=0.382)$, social jetlag $(p=0.553)$, sleep efficiency $(p=0.975)$, WASO (wakening after sleep onset; $p=0.927$ ) and total number of wake bouts $(p=0.659)$.

Measures from the non-parametric circadian rhythm analysis were analyzed across the two groups, healthy controls and ADHD participants, in a MANCOVA with chronotype as co-variate. No statistically significant effect of group was observed (Pillai's trace $=0.116 ; F=0.351$; $p=0.899$; partial ETA squared $=0.116)$. A significant difference for mid-sleep on weekend days ( $p=0.008)$, midsleep on weekdays $(p=0.001)$, but not for social jetlag $(p=0.928)$, sleep efficiency $(p=0.715)$, WASO (wakening after sleep onset; $p=0.925$ ) and total number of wake bouts $(p=0.570)$ was observed. The measurements for two ADHD volunteers were not completed.

\section{Cell viability}

The viability of the cultivated human dermal fibroblasts (HDF) after ATO incubation was compared with HDFs without ATO. The viability of cells treated with ATO
(0.20 $\mu$ M ATO: $96.02 \pm 1.23$, mean \pm SD; $0.58 \mu \mathrm{M}$ ATO: $97.03 \pm 0.24$, mean $\pm \mathrm{SD})$ was marginally decreased compared to control cells without ATO (0 $\mu \mathrm{M}$ ATO: $97.28 \pm 0.32$, mean $\pm \mathrm{SD})$.

\section{Circadian gene expression in human dermal fibroblasts}

The expression profiles of circadian genes after incubation with $0.20 \mu \mathrm{M}$ and $0.58 \mu \mathrm{M}$ ATO concentrations were examined in primary fibroblasts cultured from skin biopsies collected from ADHD and healthy participants and synchronized with dexamethasone. Cultures without ATO were used as a negative control.

Circwave analysis revealed a significant rhythmicity of Clock gene ( $p=0.019$, CircWave) in the cultures collected from the ADHD participants incubated with $0.58 \mu \mathrm{M}$ ATO. This effect was not observed in other HDF cultures.

Mixed between-within ANOVA analysis of circadian gene data with group as between-subject factor and time as within-subject factor showed significant main effects for time for all circadian genes $(p<0.01)$. Bmall expression showed a significant zeitgeber time $\mathrm{ZT} \times$ group interaction via mixed ANOVA (Greenhouse-Geisser corrected $F_{0,76.21,68}=1.716, p=0.027$, partial ETA squared $\left.=0.125\right)$, as well as Per2 expression (Greenhouse-Geisser corrected $F_{1,32.25,29}=2.308, p=0.001$, partial ETA squared $=0.168$ ). No ZT $\times$ group interaction via mixed ANOVA was observed for Clock (Greenhouse-Geisser corrected $F_{0.41,26.67}=1.256$, $p=0.183$, partial ETA squared $=0.095)$, Cryl (Greenhouse-Geisser corrected $F_{0.60,20.30}=1.335, p=0.157$, partial ETA squared $=0.103$ ), Perl (Greenhouse-Geisser corrected $F_{1.37,21.52}=1.368, p=0.131$, partial ETA squared $=0.098$ ) and Per3 (Greenhouse-Geisser corrected $F_{2.16,15.76}=1.554$, $p=0.087$, partial ETA squared $=0.118$ ) expression.

Bonferroni post hoc correction showed Bmall expression at zeitgeber time ZT16 to be significant higher in cultures incubated with $0.58 \mu \mathrm{M}$ ATO compared to those with $0.2 \mu \mathrm{M}$ ATO among healthy controls $(p=0.005)$ and $\operatorname{ADHD}(p=0.019)$. The same effects were observed in HDFs from volunteers with a diagnosis of ADHD with $0.58 \mu \mathrm{M}$ $(p=0.003)$ compared to HDFs from HCs with $0.2 \mu \mathrm{M}$ ATO. HDFs from volunteers with a diagnosis of ADHD incubated with $0.2 \mu \mathrm{M}$ ATO resulted in different Bmall expressions at ZT16 ( $p=0.030)$ and ZT28 $(p=0.019)$ compared to HC cultures incubated with $0.58 \mu \mathrm{M}$ ATO. $0.20 \mu \mathrm{M}$ ATO induced different Bmall expression between controls and ADHD group at ZT28 $(p=0.031)$. The expression of Per 2 at ZT 12 $(p=0.015)$ was significant higher in HDF cultures incubated with $0.20 \mu \mathrm{M}$ ATO compared to those exposed to $0.58 \mu \mathrm{M}$ ATO. The expression was lower at ZT4 $(p=0.029)$ for HDF cultures from HCs with $0.20 \mu \mathrm{M}$ ATO compared to ADHD participants without ATO (Figs. 1, 2, 3). 


\section{ADHD with ATO compared with HC NegControl}
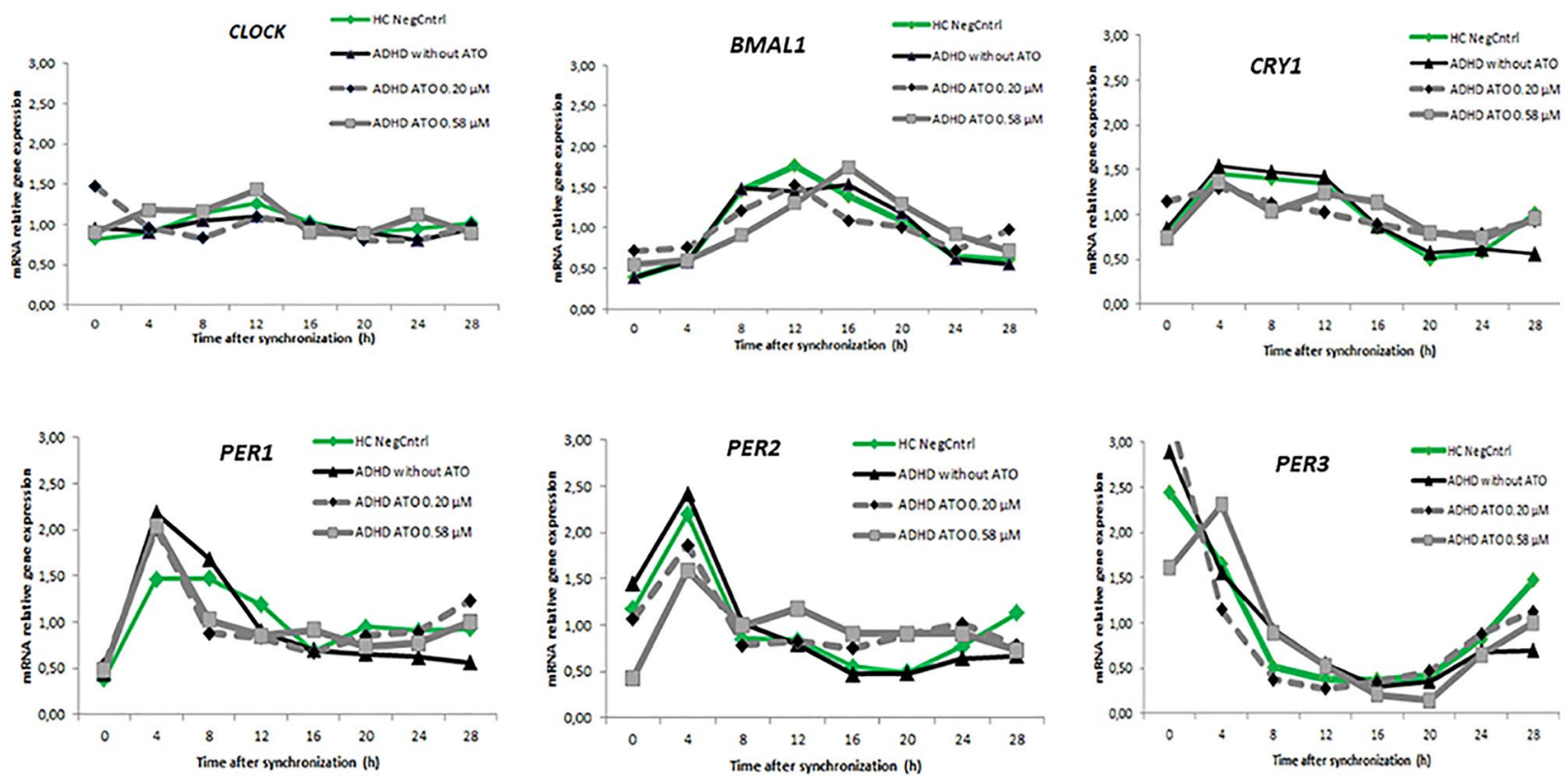

Fig. 1 Relative mRNA gene expression of circadian genes in healthy controls $(0 \mu \mathrm{M})$ and ADHD volunteers $(0,0.2,0.58 \mu \mathrm{M}$ ATO $)$

Gene expression in healthy participants revealed a statistical significant difference between cultures incubated with ATO and negative controls (without ATO incubation), as determined by one-way ANOVA for Clock at ZT16 $(F=4.108, p=0.026), C r y l$ at ZT0 $(F=5.069, p=0.012)$ and ZT28 $(F=4.006, p=0.030)$, Per2 at ZT4 $(F=4.023$, $p=0.027)$ and Per3 at ZT $12(F=6.151, p=0.005)$. A Bonferroni post hoc analysis revealed a significant lower expression in cultures incubated with $0.2 \mu \mathrm{M}$ ATO compared to negative controls for Clock (ZT16, $p=0.050)$ and Per2 (ZT4, $p=0.045)$. The same effect was observed for the cultures incubated with $0.58 \mu \mathrm{M}$ ATO for Cryl (ZT28, $p=0.036)$. In contrast to this, the expression levels of Cryl at ZT0 $(\mathrm{p}=0.012), \operatorname{Per} 2$ at ZT20 $(p=0.008)$ and Per3 at ZT12 $(p=0.017)$ were higher in HDFs incubated with ATO compared to the cultures without ATO. The Bmall $(F=8.086, p=0.001), \operatorname{Per} 1(F=4.773, p=0.015)$ and Per3 $(F=6.151, p=0.005)$ expression differed between the two ATO concentrations in HDFs from HC. This was observed particularly at ZT16 for Bmall $(p=0.001)$ and ZT12 for period genes (Perl, $p=0.012 ;$ Per3, $p=0.011)$ (Figs. 4, 5).

One-way ANOVA in the ADHD group revealed statistical significant differences between cultures incubated with ATO and negative controls for Clock $(F=6.703, p=0.004)$, Bmall (ZT16, $F=5.372, p=0.010$; ZT28, $F=3.372$, $p=0.047), \operatorname{Per} 2(F=3.335, p=0.048)$ and Per3 (ZT0,
$F=5.836, p=0.007 ; \mathrm{ZT} 8, F=2.465, p=0.027)$. A Bonferroni posthoc correction revealed a significant higher expression in cultures incubated with $0.2 \mu \mathrm{M}$ ATO compared to negative controls and those with $0.58 \mu \mathrm{M}$, particularly at ZT0 for Clock $(p=0.016)$ and Per3 $(p=0.007)$ and ZT28 for Bmall $(p=0.047)$. The expression was significant lower at ZT8 for Per3 $(p=0.043)$ and ZT16 for Bmall $(p=0.009)$ and $\operatorname{Per} 2(p=0.047)$ in cultures incubated with $0.2 \mu \mathrm{M}$ ATO compared to either negative controls or those with $0.58 \mu \mathrm{M}$ ATO (Figs. 4, 5).

\section{Chronotype and circadian gene expression}

$58.3 \%$ of healthy participants displayed neutral preference, whereas $25.0 \%$ had moderate morning and $16.7 \%$ definite morning preference. $41.7 \%$ of ADHD participants displayed moderate morning and $25.0 \%$ neutral preference. Interestingly, in the ADHD group, the evening preference was represented by $16.7 \%$ definite evening and $16.7 \%$ moderate evening chronotype. There were no participants with definitive morning preference in the ADHD group.

The relationship between chronotype and circadian gene relative expressions was determined using Spearman's rank order correlation. Strong positive correlations for chronotype and circadian genes were observed for Bmall, Per3 and Cryl among the study participants. 
BMAL1

\section{ZT $\times$ group interaction via mixed ANOVA}

Greenhouse-Geisser corrected : $F_{0,76.21,68}=1,716, P=0,027$, partial ETA squared $=0.125$
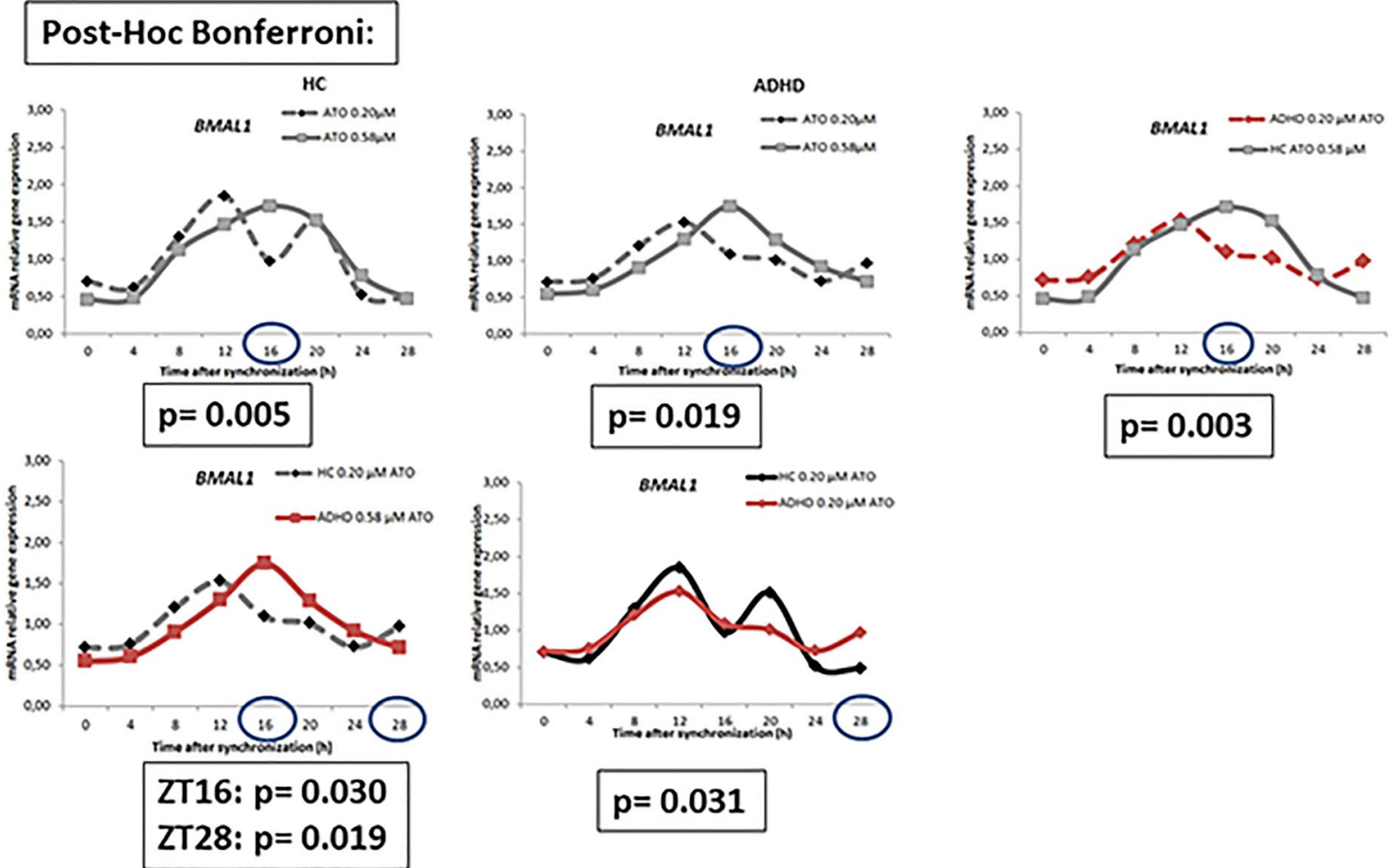

Fig. 2 Relative mRNA gene expression of Bmal1 in healthy controls and ADHD volunteers $(0,0.2,0.58 \mu \mathrm{M}$ ATO $)$

In ADHD group chronotype and Bmall gene were positively correlated in the first $8 \mathrm{~h}$ after dexamethasone synchronization $\left(\mathrm{ZT} 4, r_{s}=0.649, p=0.022 ; \mathrm{ZT} 8, r_{s}=0.847\right.$, $p=0.001)$. In ADHD cultures incubated with $0.58 \mu \mathrm{M}$ ATO, a positive correlation between chronotype and Per3 at ZT0 $\left(r_{s}=0.590, p=0.043\right)$ was observed. Additionally, in healthy participants group was a strong positive correlation for chronotype and clock genes, particularly at ZT0 for Per3 gene $\left(r_{s}=0.631, p=0.028\right)$, and ZT16 for Bmall $\left(r_{s}=0.611, p=0.035\right)$ as well as Cryl $\left(r_{s}=0.591\right.$, $p=0.043)$, respectively. In HC cultures incubated with $0.58 \mu \mathrm{M}$ ATO, the strong positive correlation for chronotype with Bmall was shifted to ZT20 $\left(r_{s}=0.650\right.$, $p=0.022$ ).

Differences of clock gene expression levels among chronotype were assessed using one-way ANOVA. Gene expression in HDFs from healthy participants revealed a statistical significant difference between chronotypes for Per3 immediately after synchronization at ZT0 $(F=6.804$, $p=0.016)$ and $16 \mathrm{~h}$ after synchronization for Bmall $(F=4.884, p=0.037)$. A Bonferroni post hoc correction revealed a significant lower expression of $\operatorname{Per} 3(p=0.018)$ and Bmall $(p=0.040)$ in healthy participants exhibiting neutral chronotype compared to those with moderate morning type. One-way ANOVA in the ADHD group revealed no statistical significant clock genes differences between chronotypes.

Exposure of HDFs cultures with ATO revealed differences between chronotypes. In HC cultures incubated with $0.58 \mu \mathrm{M}$ ATO, one-way ANOVA revealed a statistical significant gene expression differences between chronotypes for Bmall (ZT0, $F=4.822, p=0.038$; ZT20, $F=9.168$, $p=0.007), \operatorname{Clock}(F=4.491, p=0.044)$ and Per2 $(F=7.039$, $p=0.014)$. Comparing healthy participants with definitive morning to those with moderate morning preference, Bonferroni post hoc correction showed significant lower Bmall expression levels at ZT8 $(p=0.038)$ and ZT20 $(p=0.044)$. The expression of Clock at ZT24 $(p=0.047)$ was significant higher among the healthy participants with moderate morning compared to those exhibiting definitive morning preference. When compared to neutral type the expression of Bmall at ZT20 $(p=0.006)$ and Per2 at ZT28 $(p=0.014)$ was significant different in healthy participants with definitive morning, and moderate morning chronotype, respectively. 
PER2

\section{$\mathrm{ZT} \times$ group interaction via mixed ANOVA}

Greenhouse-Geisser corrected : $F_{1,32.25,29}=2,308, P=0,001$, partial ETA squared $=0.168$
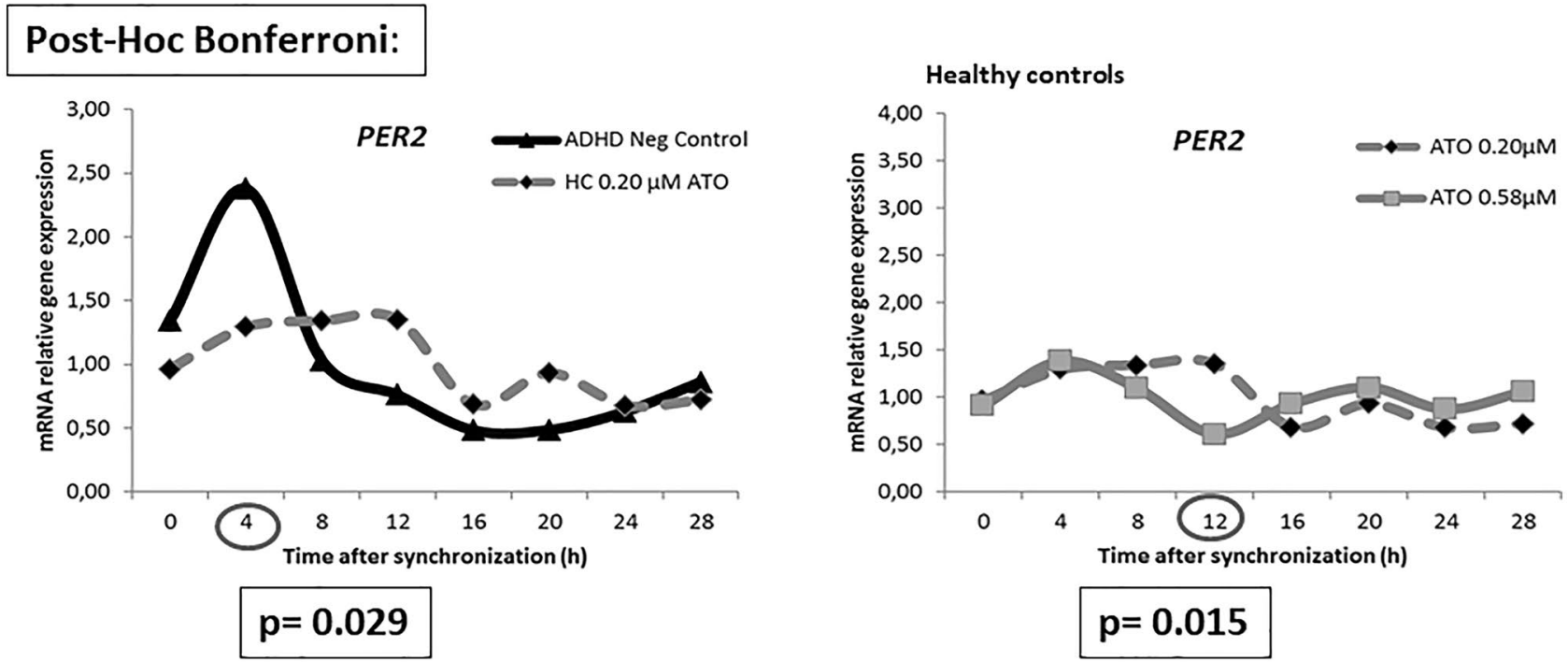

Fig. 3 Relative gene expression of Per2 in healthy controls and ADHD volunteers $(0,0.2,0.58 \mu \mathrm{M}$ ATO)

In ADHD cultures incubated with $0.20 \mu \mathrm{M}$ ATO, one-way ANOVA revealed a statistical significant differences in gene expression between chronotypes for Perl $(F=8.090$, $p=0.008)$ and Per2 $(F=5.278, p=0.027)$. Bonferroni post hoc correction showed that the participants with definite evening preference had a higher Per1 at ZT8 compared to ADHD participants with neutral $(p=0.013)$ and moderate morning preference $(p=0.015)$. Similarly, different Per2 expressions levels were observed in ADHD participants exhibiting evening preferences at ZT4 $(p=0.025)$. Additionally, in participants with moderate evening preference, the expression of Per2 at ZT20 was significant higher compared to those exhibiting definitive evening $(p=0.012)$, neutral $(p=0.038)$ and moderate morning preference $(p=0.046)$. Higher concentration of ATO induced higher Bmall expression levels $(F=5.160, p=0.028)$ in HDF cultures from ADHD participants with moderate evening chronotype, particularly at ZT12 after synchronization (Bonferroni post hoc test, $p=0.041$ ) compared to the participants with definite evening type.

When comparing the two study groups, significant different Clock $(F=4.145, p=0.010)$, Bmall $(F=4.815$, $p=0.005)$ and $\operatorname{Per} 3(F=4.042, p=0.011)$ expressions were observed in HDFs exposed to ATO collected from ADHD participants exhibiting neutral and moderate evening preference, as well as from healthy participants with morning preference. ADHD participants with moderate evening chronotype presented significant lower Clock $(0.20 \mu \mathrm{M}$ ATO, ZT16,
Bonferroni post hoc test, $p=0.007)$ and Bmall $(0.58 \mu \mathrm{M}$ ATO, ZT20, Bonferroni post hoc test, $p=0.015$ ) expression levels compared to $\mathrm{HC}$ with definitive morning and moderate morning chronotype, respectively. ADHD participants with neutral type revealed higher Per 3 expression $(0.58 \mu \mathrm{M}$ ATO, ZT28, Bonferroni post hoc test, $p=0.011$ ) than HC with moderate morning chronotype.

\section{Discussion}

The results of the present study illustrate that attention-deficit hyperactivity disorder (ADHD) are associated to alterations in the circadian rhythm. It demonstrates that atomoxetine (ATO) impacts on circadian function, particularly the Clock, Bmall and Per2 gene expression.

Ex vivo expression of circadian genes in fibroblasts has previously been demonstrated to be a useful approach to study circadian rhythms related to sleep/wake behavior (Brown 2005; Hida 2017) in psychiatric disorders and particularly ADHD (Coogan et al. 2019; Johansson et al. 2011, 2016). ADHD is frequently linked with sleep disorders such as obstructive sleep apnea, peripheral limb movement disorder, restless legs syndrome and circadian-rhythm sleep disorders (Hvolby 2015).

Previously, we reported that patients with ADHD using ADHD-medication, such as methylphenidate and ATO, have altered sleep activity compared to both controls and ADHD 


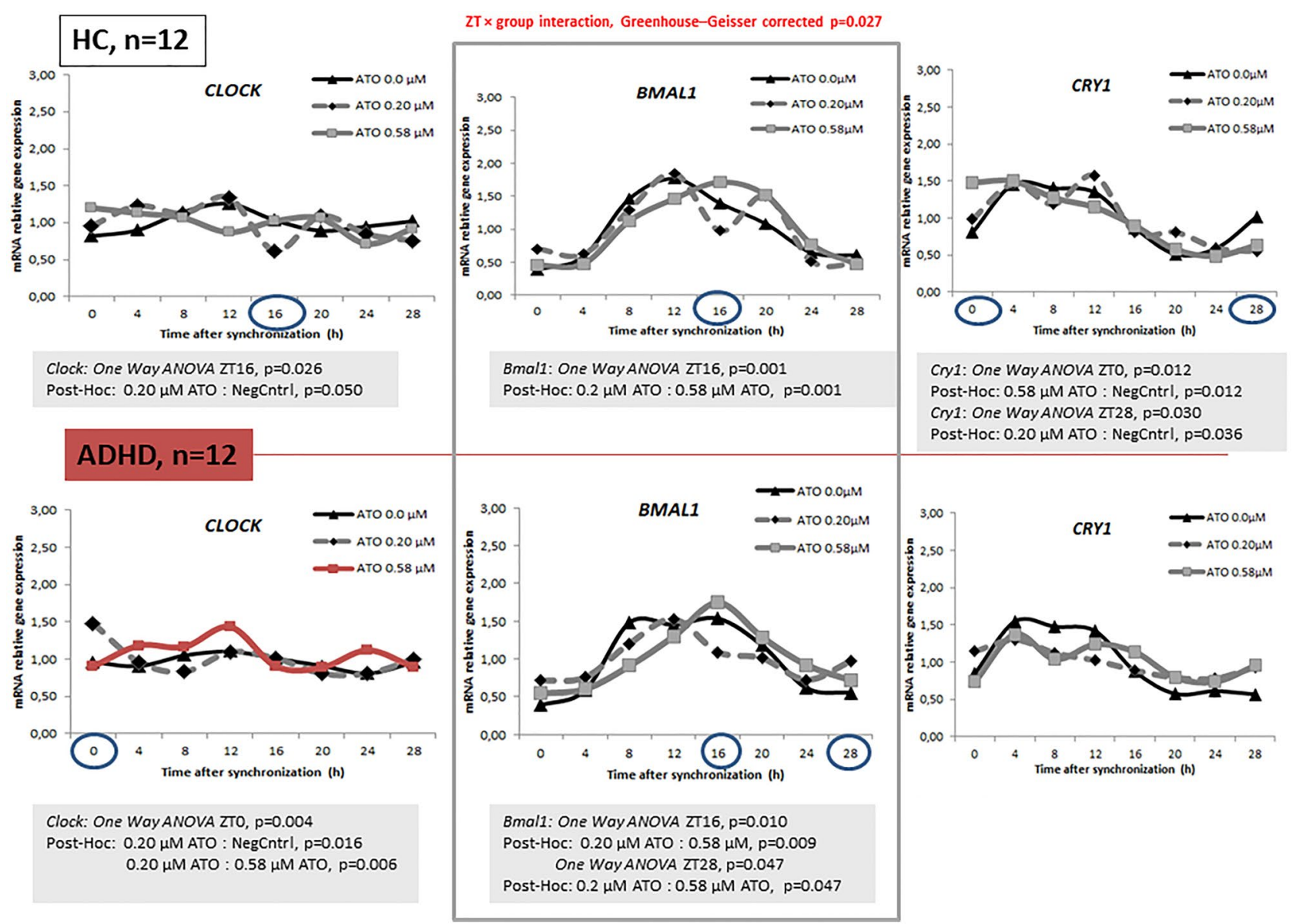

Fig. 4 Relative mRNA gene expression of circadian genes (Clock, Bmall, Cry1) in healthy controls and ADHD volunteers $(0,0.2,0.58 \mu \mathrm{M}$ ATO)

participants without medication (Coogan et al. 2019). In the current study, measures from the non-parametric circadian rhythm analysis between both groups, healthy controls and ADHD participant showed no statistical significant effect of group for mid-sleep on weekend days, mid-sleep on weekdays, social jetlag, sleep WASO and total number of wake bouts.

ADHD individuals without medication showed alterations in the expression of Per2 and Cryl compared to medicated ADHD patients or HC. Clock expression was altered in patients with ADHD using methylphenidate and ATO, but showed no significant rhythmicity (Coogan et al. 2019).

The incubation of HDF cultures collected from ADHD participants to $0.58 \mu \mathrm{M}$ ATO, induced the rhythmicity of Clock, whereas this effect was not observed in other HDF cultures. It is to mention that previously several studies reported that Clock features minimal circadian variation. No significant $24 \mathrm{~h}$ variation in Clock expression was observed in oral mucosa and skin biopsies obtained from healthy participants. Clock was found to be arrhythmic in mice liver (Bjarnason 2001; Bonaconsa et al. 2014). Hansen et al.
(2016) investigated if rhythmicity of clock- and metabolic gene expression is altered in human primary myotubes. The study groups were represented by type 2 diabetic patients with BMI- and age-matched obese controls, and lean, healthy, young endurance trained athletes with their agematched sedentary controls. Hansen et al. (2016) confirmed no circadian rhythmicity for $C l o c k$ gene expression whereas Bmall, Cryl, Per2 and Per3 showed significant circadian rhythmicity in the study groups (Hansen et al. 2016).

In the present study, the observed ATO effect in the ADHD group on Clock rhythmicity could suggest a potential link between the norepinephrine and circadian rhythm pathways. ATO, as a selective NET inhibitor, modulates norepinephrine. Several studies have shown that norepinephrine is a synchronizer of the circadian rhythm (Chalmers et al. 2008; Li and Cassone 2015; Maletic et al. 2017; Morioka et al. 2010). Moreover, $48 \mathrm{~h}$ norepinephrine synchronization of pineal gland cultures from rats, induced a rhythmic expression pattern of the clock genes (Andrade-Silva et al. 2014). Preliminary results from our studies show that Clock gene rhythmicity is linked to higher body mass index (BMI). 


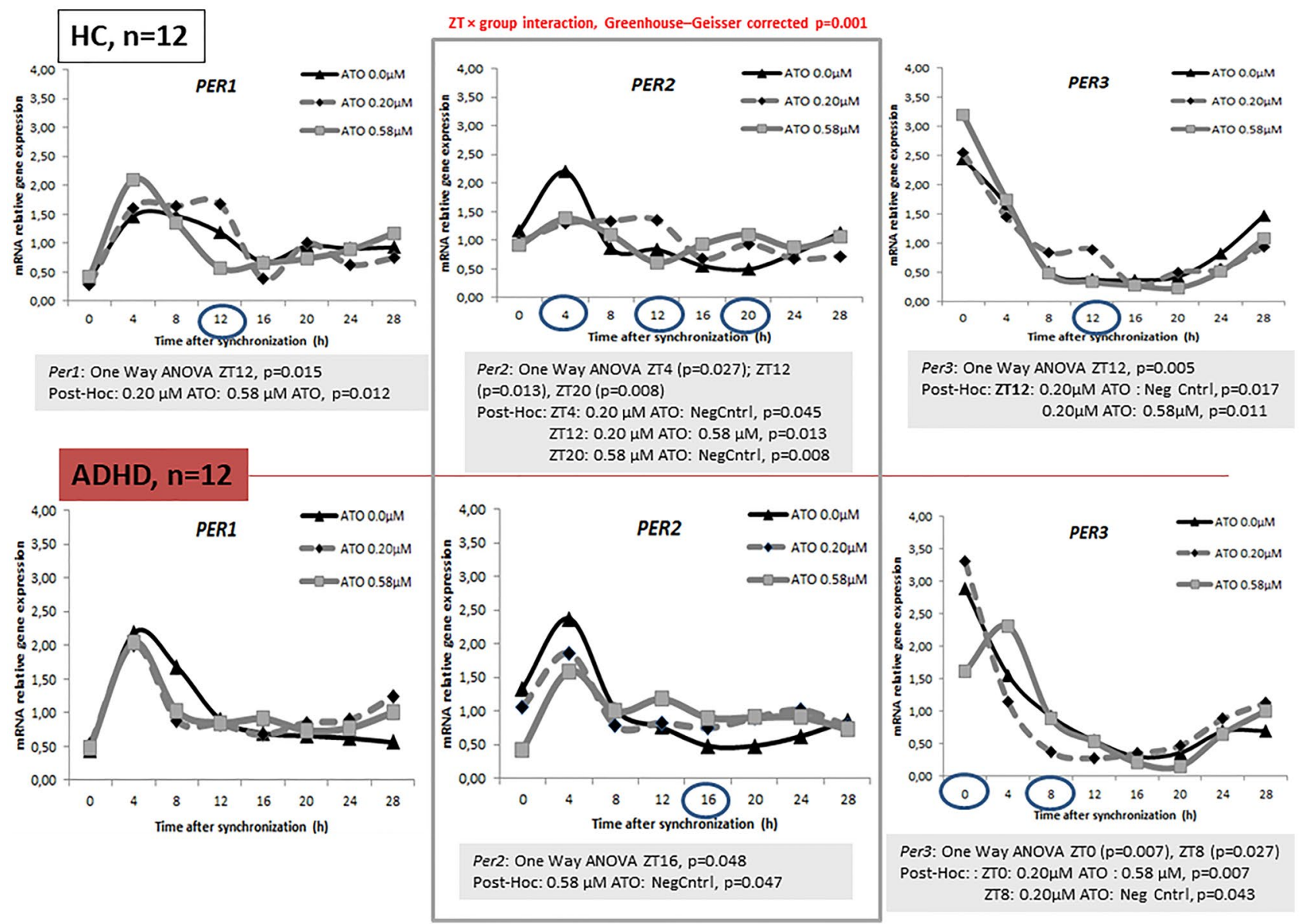

Fig. 5 Relative mRNA gene expression of circadian genes (Per1-3) in healthy controls and ADHD volunteers $(0,0.2,0.58 \mu \mathrm{M}$ ATO)

In the present study, there were no significant differences in BMI values across the two study groups.

Cultures from HC exposed to $0.20 \mu \mathrm{M}$ ATO had a higher Perl and Per 3 expression, $12 \mathrm{~h}$ after dexamethasone synchronization. Paradoxically, the expression of the two period genes was lower in cultures exposed to higher ATO concentration. This observations suggests that lower dose of ATO is inducing a phase delay of the Perl and Per3 gene expression, as well as for Cryl. However, this effect was not observed in the cultures derived from ADHD participants. The expression and rhythm of all genes except Cryl in the ADHD group adjusted to the healthy control group after ATO incubation. This effect is observed particularly for the lower ATO concentration. Higher concentration of ATO adjusts the expression of Clock gene toward the HC group, and shifts the expression of Bmall and alters the expression of Per3.

We observed changes in the rhythmic expression of Bmall and Per2 genes. In ADHD patients and HC, Bmall expression differed between the two ATO concentrations 16 and $28 \mathrm{~h}$ after dexamethasone synchronization. Moreover, $0.20 \mu \mathrm{M}$ ATO induced different Bmall expression between controls and ADHD group at ZT28. In HC, the expression of Per2 at ZT12 was significant higher in HDF cultures incubated with $0.20 \mu \mathrm{M}$ ATO compared to those exposed to $0.58 \mu \mathrm{M}$ ATO. The expression of Per 2 was lower at ZT4 compared to negative control cultures from ADHD participants.

O'Keeffe et al. (2012) reported that ATO treatment at circadian time ZT6 induced a downregulation of CLOCK in the suprachiasmatic nucleus, but did not alter the expression of PER2 and BMAL1 (O'Keeffe et al. 2012).

Single nucleotide polymorphisms (SNP) in circadian genes were associated with core ADHD symptoms, increased evening-orientation and frequent sleep problem (Korman et al. 2018). Particularly Per3 gene has been associated with chronotype, sleep homeostasis and various psychiatric disorders (Archer et al. 2018; Liberman et al. 2018). However, several studies report no association between the Per3 VNTR and Clock SNP, diurnal preference and sleep/ wake behavior (An 2014; Hida 2018; McGowan et al. 2020).

A limitation of the study is the small sample size. Further studies with a higher number of participants are necessary. 
It is to mention, that no special cognitive testing was implemented in this study. In addition, the participants of the ADHD group took no medication before and during the study. For further studies, a connection between circadian disturbances, cognitive deficits and the effect of medication would be suitable.

In the present study, strong positive correlations for chronotype and clock genes were observed for Bmall, Per3 and Cryl among the study participants, particularly after atomoxetine incubation at ZT0 and ZT16. Comparing the circadian preferences, statistical significant different Clock, Bmall and Per 3 expressions were observed in HDFs exposed to ATO in ADHD participants exhibiting neutral and moderate evening preference, as well in healthy participants with morning preferences. ADHD participants with evening preference have higher Per 2 expression 4 and $20 \mathrm{~h}$ after dexamethasone synchronization compared to the other chronotypes. We also observed an overexpression for Clock $16 \mathrm{~h}$ after synchronization for ADHD participants with moderate evening compared to $\mathrm{HC}$ with definitive morning preference. In summary, our results suggest that ATO influences circadian gene expression.

Acknowledgements 2020 CoCA ADHD Comorbidity. This project has received funding from the European Union's Horizon 2020 research and innovation programme under grant agreement No 667302.

Funding Open Access funding enabled and organized by Projekt DEAL. 2020 CoCA ADHD Comorbidity. This project has received funding from the European Union's Horizon 2020 research and innovation Programme Under Grant Agreement No 667302.

Availability of data and material Data and material are available.

Code availability Actiwatch 2, Philips Respironics, USA; CircWave v. 1.4 software (generated by Dr. Roelof Hut; www.euclock.org); SPSS (IBM Corporation)

\section{Declarations}

Conflict of interest Johannes Thome has received financial support from pharmaceutical companies (Actelion, Astra Zeneca, BristolMyers Squibb, EVER Neuro Pharma GmbH, Janssen-Cilag, Lilly, Lundbeck, MEDICE, Merz, Novartis, Pfizer, Roche, Servier, Shire, Trommsdorff) some of which manufacture medication used in the treatment of ADHD patients. Frank Faltraco, Oliver Tucha, Frederick Simon, Adriana Uzoni and Denise Palm have no potential conflicts of interest to disclose.

Ethics approval Ethical approval for the conduct of the study, including obtaining human dermal biopsy samples, was given by the ethical review committee of Rostock University (Registration-Number: A2013-159).

Consent to participate Written consent was obtained from each study participant.

Consent for publication Written consent was obtained from each study participant.
Open Access This article is licensed under a Creative Commons Attribution 4.0 International License, which permits use, sharing, adaptation, distribution and reproduction in any medium or format, as long as you give appropriate credit to the original author(s) and the source, provide a link to the Creative Commons licence, and indicate if changes were made. The images or other third party material in this article are included in the article's Creative Commons licence, unless indicated otherwise in a credit line to the material. If material is not included in the article's Creative Commons licence and your intended use is not permitted by statutory regulation or exceeds the permitted use, you will need to obtain permission directly from the copyright holder. To view a copy of this licence, visit http://creativecommons.org/licenses/by/4.0/.

\section{References}

An $\mathrm{H}$ et al (2014) Chronotype and a PERIOD3 variable number tandem repeat polymorphism in Han Chinese pilots. Int J Clin Exp Med 7:3770-3776

Andrade-Silva J, Cipolla-Neto J, Peliciari-Garcia RA (2014) The in vitro maintenance of clock genes expression within the rat pineal gland under standard and norepinephrine-synchronized stimulation. Neurosci Res 81-82:1-10. https://doi.org/10.1016/j. neures.2014.03.005

Archer SN, Schmidt C, Vandewalle G, Dijk DJ (2018) Phenotyping of PER3 variants reveals widespread effects on circadian preference, sleep regulation, and health. Sleep Med Rev 40:109-126. https://doi.org/10.1016/j.smrv.2017.10.008

Baird AL, Coogan AN, Kaufling J, Barrot M, Thome J (2013) Daily methylphenidate and atomoxetine treatment impacts on clock gene protein expression in the mouse brain. Brain Res 1513:6171. https://doi.org/10.1016/j.brainres.2013.03.038

Biederman J, Mick E, Faraone SV (2000) Age-dependent decline of symptoms of attention deficit hyperactivity disorder: impact of remission definition and symptom type. Am J Psychiatry 157:816-818. https://doi.org/10.1176/appi.ajp.157.5.816

Bjarnason GA et al (2001) Circadian expression of clock genes in human oral mucosa and skin: association with specific cellcycle phases. Am J Pathol 158:1793-1801. https://doi.org/10. 1016/S0002-9440(10)64135-1

Bonaconsa M, Malpeli G, Montaruli A, Carandente F, Grassi-Zucconi G, Bentivoglio M (2014) Differential modulation of clock gene expression in the suprachiasmatic nucleus, liver and heart of aged mice. Exp Gerontol 55:70-79. https://doi.org/10.1016/j. exger.2014.03.011

Brown SA et al (2005) The period length of fibroblast circadian gene expression varies widely among human individuals. PLoS Biol 3:e338. https://doi.org/10.1371/journal.pbio.0030338

Bymaster FPK, Nelson DL, Hemrick-Luecke SK, Threlkeld PG, Heiligenstein JH, Morin SM, Gehlert DR, Perry KW (2002) Atomoxetine increases extracellular levels of norepinephrine and dopamine in prefrontal cortex of rat. Neuropsychopharmacology 27:699-711

Chalmers JA, Martino TA, Tata N, Ralph MR, Sole MJ, Belsham DD (2008) Vascular circadian rhythms in a mouse vascular smooth muscle cell line (Movas-1). Am J Physiol Regul Integr Comp Physiol 295:R1529-R1538. https://doi.org/10.1152/ajpregu. 90572.2008

Coogan AN et al (2019) Impact of adult attention deficit hyperactivity disorder and medication status on sleep/wake behavior and molecular circadian rhythms. Neuropsychopharmacology 44:1198-1206. https://doi.org/10.1038/s41386-019-0327-6

Faltraco F, Palm D, Uzoni A, Borchert L, Simon F, Tucha O, Thome J (2021a) Dopamine adjusts the circadian gene expression 
of Per2 and Per3 in human dermal fibroblasts from ADHD patients. J Neural Transm. https://doi.org/10.1007/s00702-02102374-4 (accepted)

Faltraco F, Palm D, Coogan A, Uzoni A, Duwe I, Simon F, Tucha O, Thome J (2021b) Remdesivir shifts circadian rhythmicity to eveningness; similar to the most prevalent chronotype to ADHD. J Neural Transm. https://doi.org/10.1007/s00702-02102375-3 (accepted)

Gaspar L et al (2017) The genomic landscape of human cellular circadian variation points to a novel role for the signalosome. Elife. https://doi.org/10.7554/eLife.24994

Gerhart-Hines Z, Lazar MA (2015) Circadian metabolism in the light of evolution. Endocr Rev 36:289-304. https://doi.org/10. 1210/er.2015-1007

Griffiths KR et al (2018) Response inhibition and emotional cognition improved by atomoxetine in children and adolescents with ADHD: the ACTION randomized controlled trial. J Psychiatr Res 102:57-64. https://doi.org/10.1016/j.jpsychires.2018.03. 009

Hansen J, Timmers S, Moonen-Kornips E, Duez H, Staels B, Hesselink MK, Schrauwen P (2016) Synchronized human skeletal myotubes of lean, obese and type 2 diabetic patients maintain circadian oscillation of clock genes. Sci Rep 6:35047. https:// doi.org/10.1038/srep35047

Hida A et al (2018) Lack of association between PER3 variable number tandem repeat and circadian rhythm sleep-wake disorders. Hum Genome Var 5:17. https://doi.org/10.1038/ s41439-018-0017-7

Hida A et al (2017) Evaluation of circadian phenotypes utilizing fibroblasts from patients with circadian rhythm sleep disorders Transl. Psychiatry 7:e1106. https://doi.org/10.1038/tp.2017.75

Hu Y, Shmygelska A, Tran D, Eriksson N, Tung JY, Hinds DA (2016) GWAS of 89,283 individuals identifies genetic variants associated with self-reporting of being a morning person. Nat Commun 7:10448. https://doi.org/10.1038/ncomms 10448

Hvolby A (2015) Associations of sleep disturbance with ADHD: implications for treatment. Atten Defic Hyperact Disord 7:1-18. https:// doi.org/10.1007/s12402-014-0151-0

Johansson AS, Owe-Larsson B, Hetta J, Lundkvist GB (2016) Altered circadian clock gene expression in patients with schizophrenia. Schizophr Res 174:17-23. https://doi.org/10.1016/j.schres.2016. 04.029

Johansson J, Landgren M, Fernell E, Vumma R, Ahlin A, Bjerkenstedt L, Venizelos N (2011) Altered tryptophan and alanine transport in fibroblasts from boys with attention-deficit/hyperactivity disorder (ADHD): an in vitro study. Behav Brain Funct 7:40. https://doi. org/10.1186/1744-9081-7-40

Jones SE et al (2016) Genome-wide association analyses in 128,266 individuals identifies new morningness and sleep duration loci. PLoS Genet 12:e1006125. https://doi.org/10.1371/journal.pgen. 1006125

Kalmbach DA, Schneider LD, Cheung J, Bertrand SJ, Kariharan T, Pack AI, Gehrman PR (2017) Genetic basis of chronotype in humans: insights from three landmark GWAS. Sleep. https://doi. org/10.1093/sleep/zsw048

Konofal E, Lecendreux M, Cortese S (2010) Sleep and ADHD. Sleep Med 11:652-658. https://doi.org/10.1016/j.sleep.2010.02.012

Korman M, Palm D, Uzoni A, Faltraco F, Tucha O, Thome J, Coogan AN (2018) ADHD 24/7: circadian clock genes, chronotherapy and sleep/wake cycle insufficiencies in ADHD. World J Biol Psychiatry. https://doi.org/10.1080/15622975.2018.1523565

Lane JM et al (2016) Genome-wide association analysis identifies novel loci for chronotype in 100,420 individuals from the UK Biobank. Nat Commun 7:10889. https://doi.org/10.1038/ncomm s10889
Li Y, Cassone VM (2015) Clock-controlled regulation of the acute effects of norepinephrine on chick pineal melatonin rhythms. J Biol Rhythms 30:519-532. https://doi.org/10.1177/0748730415 607060

Liberman AR, Halitjaha L, Ay A, Ingram KK (2018) Modeling strengthens molecular link between circadian polymorphisms and major mood disorders. J Biol Rhythms 33:318-336. https://doi. org/10.1177/0748730418764540

Livak KJ, Schmittgen TD (2001) Analysis of relative gene expression data using real-time quantitative PCR and the 2(-Delta Delta C(T)) method. Methods 25:402-408. https://doi.org/10.1006/ meth.2001.1262

Maletic V, Eramo A, Gwin K, Offord SJ, Duffy RA (2017) The role of norepinephrine and its $\alpha$-adrenergic receptors in the pathophysiology and treatment of major depressive disorder and schizophrenia: a systematic review front. Psychiatry 8:42. https://doi.org/10 3389/fpsyt.2017.00042

Mane VP, Heuer MA, Hillyer P, Navarro MB, Rabin RL (2008) Systematic method for determining an ideal housekeeping gene for real-time PCR analysis. J Biomol Tech 19:342-347

McGowan NM, Uzoni A, Faltraco F, Thome J, Coogan AN (2020) The impact of social jetlag and chronotype on attention, inhibition and decision making in healthy adults. J Sleep Res. https://doi.org/10. $1111 /$ jsr. 12974

Menet JS, Rodriguez J, Abruzzi KC, Rosbash M (2012) Nascent-seq reveals novel features of mouse circadian transcriptional regulation. Elife 1:e00011. https://doi.org/10.7554/eLife.00011

Michelson DF, Wernicke J, Kelsey D, Kendrick K, Sallee FR, Spencer $\mathrm{T}$ (2001) Atomoxetien in the treatment of children and adolescents with attention-deficit/hyperactivity disorder. Pediatrics 108(5):e83

Morioka N, Sugimoto T, Tokuhara M, Dohi T, Nakata Y (2010) Noradrenaline induces clock gene Per1 mRNA expression in C6 glioma cells through $\beta 2$-adrenergic receptor coupled with protein kinase A-cAMP response element binding protein (PKACREB) and Src-Tyrosine Kinase-Glycogen Synthase Kinase-3 $\beta$ (Src-GSK-3 $\beta$ ). J Pharmacol Sci 113:234-245. https://doi.org/10. 1254/jphs.10031FP

O'Keeffe SM, Thome J, Coogan AN (2012) The noradrenaline reuptake inhibitor atomoxetine phase-shifts the circadian clock in mice. Neuroscience 201:219-230. https://doi.org/10.1016/j.neuro science.2011.11.002

Palm D, Uzoni A, Simon F, Tucha F, Thome J, Faltraco F (2021) Norepinephrine influences the circadian clock in human dermal fibroblasts from participants with a diagnosis of attention deficit hyperactivity disorder. J Neural Transm. https://doi.org/10.1007/ s00702-021-02376-2 (accepted)

Philipsen A, Hornyak M, Riemann D (2006) Sleep and sleep disorders in adults with attention deficit/hyperactivity disorder. Sleep Med Rev 10:399-405. https://doi.org/10.1016/j.smrv.2006.05.002

Rybak YE, McNeely HE, Mackenzie BE, Jain UR, Levitan RD (2007) Seasonality and circadian preference in adult attention-deficit/ hyperactivity disorder: clinical and neuropsychological correlates. Compr Psychiatry 48:562-571. https://doi.org/10.1016/j.compp sych.2007.05.008

Spencer T et al (2001) An open-label, dose-ranging study of atomoxetine in children with attention deficit hyperactivity disorder. J Child Adolesc Psychopharmacol 11:251-265. https://doi.org/10. $1089 / 10445460152595577$

Strober W (2015) Trypan blue exclusion test of cell viability. Curr Protoc Immunol 111:A3B1-A3B3. https://doi.org/10.1002/04711 42735.ima03bs111

Suzuki C, Ikeda Y, Tateno A, Okubo Y, Fukayama H, Suzuki H (2019) Acute atomoxetine selectively modulates encoding of reward value in ventral medial prefrontal cortex. J Nippon Med Sch 86:98-107. https://doi.org/10.1272/jnms.JNMS.2019_86-205 
Swanson CJ, Perry KW, Koch-Krueger S, Katner J, Svensson KA, Bymaster FP (2006) Effect of the attention deficit/hyperactivity disorder drug atomoxetine on extracellular concentrations of norepinephrine and dopamine in several brain regions of the rat. Neuropharmacology 50:755-760. https://doi.org/10.1016/j.neuro pharm.2005.11.022

Takashima A (1998) Establishment of fibroblast cultures. Curr Protoc Cell Biol. https://doi.org/10.1002/0471143030.cb0201s00
Thaben PF, Westermark PO (2016) Differential rhythmicity: detecting altered rhythmicity in biological data. Bioinformatics 32:2800 2808. https://doi.org/10.1093/bioinformatics/btw309

Publisher's Note Springer Nature remains neutral with regard to jurisdictional claims in published maps and institutional affiliations. 